\title{
Inventory and Production Dynamics in a Discrete-Time Vendor-Managed Inventory Supply Chain System
}

\author{
Yongchang Wei $\mathbb{D}^{1},{ }^{1}$ Fangyu Chen $\left(\mathbb{D},{ }^{1}\right.$ and Hongwei Wang $\mathbb{D}^{2,3}$ \\ ${ }^{1}$ School of Business Administration, Zhongnan University of Economics and Law, Wuhan, China \\ ${ }^{2}$ School of Management, Huazhong University of Science and Technology, Wuhan, China \\ ${ }^{3}$ School of Automation, Huazhong University of Science and Technology, Wuhan, China \\ Correspondence should be addressed to Hongwei Wang; hwwang@hust.edu.cn
}

Received 18 June 2018; Accepted 1 September 2018; Published 19 September 2018

Academic Editor: Lu Zhen

Copyright (C) 2018 Yongchang Wei et al. This is an open access article distributed under the Creative Commons Attribution License, which permits unrestricted use, distribution, and reproduction in any medium, provided the original work is properly cited.

\begin{abstract}
This paper presents some analytical results on production and order dynamics in the context of a discrete-time VMI supply chain system composed of one retailer and one manufacturer. We firstly derive the lower bound and upper bound on the range of inventory fluctuations for the retailer under unknown demand. We prove that the production fluctuations can be interestingly smoothed and stabilized independent of the delivery frequency of the manufacturer used to satisfy the retailer's demand, even if the retailer subsystem is unstable. The sufficient and necessary stability condition for the whole supply chain system is obtained. To further explore the production fluctuation problem, the bullwhip effect under unknown demand is explored based on a transfer function model with the purpose of disclosing the influences of parameters on production fluctuations. Finally, simulation experiments are used to validate the theoretical results with respect to inventory and production fluctuations.
\end{abstract}

\section{Introduction}

Vendor-managed inventory (VMI) is a well-known collaborative program [1-3], in which a retailer propagates inventory information and point of sales data to its supplier, while the upstream supplier makes replenishment decision for both the retailer and itself. Since it was pioneered by Wal-Mart in the early 1980s [4], VMI has been successfully implemented by many leading firms from different industries, such as Glaxosmithkline [5], Nestle and Tesco [6], Boeing and Alcoa [7], and Shell Chemical [8].

The benefits of VMI for both suppliers and retailers have been extensively studied in the literature [9-11]. For vendors, the VMI program improves decision-making flexibility since they gain the right to determine the amount and timing of the order for retailers, which further leads to reduced bullwhip effect [12] and better utilization of manufacturing capacity, as well as better synchronization of replenishment planning [13]. For retailers, VMI saves ordering cost and inventory cost and ensures the customer service level through signing VMI contracts. For example, a supplier will be penalized once the inventory of retailer exceeds certain ranges [14]. To successfully implement the VMI program, how to make use of the flexibility of decision-making in inventory control to benefit both upstream and downstream members is a highly challenging problem.

Most of the existing literature has focused on game theory or optimization models [15-19], while this paper explores the impact of replenishment parameters on the inventory and production dynamics [20], which are closely related to the total costs. Significant costs can be incurred by production fluctuations due to frequently switching production quantities up and down, which further complicate the activities in labor force arrangement, capacity adjustment, and equipment maintenance and management. However, in a dynamic system with uncertain demand, deriving optimal solutions from an optimization model incorporating all these cost components is impractical. Furthermore, in practice, through information sharing even with order batching [21-24], VMI has become one of the frequently employed programs for eliminating bullwhip effect $[23,25,26]$. This implies that production can be smoothed and transportation cost can be 
saved via the implementation of VMI program. However, we notice that the majority of existing bullwhip literature has ignored the interaction between inventory and production fluctuations.

In a VMI system, the upstream supplier has the flexibility to determine the amount and timing of the replenishment order for the retailer. However, as mentioned, the supplier might be penalized once the retailer's inventory exceeds the predetermined range. Therefore, in order to reduce penalty cost, the supplier should know how the replenishment parameters for the retailer affect the range of inventory fluctuations of the retailer. In addition, the supplier should also consider the production or ordering cost and inventory cost for the whole system when designing its own replenishment policies. In this paper, we aim to answer three questions for the implementation of VMI: (1) How the replenishment rules designed by the supplier for itself and retailer affect the stability, inventory fluctuations, and production smoothing for the entire system under uncertain demand? (2) What is the trade-off between inventory fluctuations and production fluctuations? Specifically, what actions can be taken to limit inventory and order fluctuations. This question will be addressed in terms of stability. As investigated in the literature, stability is a fundamental problem for any dynamical system including supply chain systems [21, 27, 28]. After being disrupted, the state of a stable system will return to its steady state gradually. Actually, a stable supply chain system tends to be resilient in the presence of demand disruptions [29]. Furthermore, stability analysis simplifies the parameters selection and performance optimization. For a stable supply chain system, stability limits the fluctuation of inventory and order in a certain range after the sudden change of demand. By contrast, unstable designs will cause undesirable fluctuations for order and inventory, which leads to high cost due to inventory accumulation or stockouts. (3) How the replenishment parameters affect the magnitude of production smoothing for the manufacturer.

Specifically, we attempt to explore the inventory and production fluctuations in the context of a VMI supply chain system composed of a manufacturer and a retailer. The manufacturer uses a reorder point policy to manage the retailer's inventory and the APIOBPCS (automatic pipeline, inventory, and order based production control system) for production control $[12,30]$. The contributions of this paper lie in threefold. Firstly, we analytically studied the impact of reorder point, delivery frequency, lead time, and demand characteristics on the range of the retailer's inventory under uncertain demand by extending a more generalized replenishment policy. Secondly, we derived the necessary and sufficient stability conditions of the VMI system for arbitrary lead times by considering the interaction between the retailer subsystem and the manufacturer subsystem, which are significant due to the development of global supply chains and complicated manufacturing process [31]. We prove that production fluctuations are independent of the replenishment parameters in the reorder point policy. Besides, we demonstrate that although the production of the upstream manufacturer can be perfectly smoothed without paying extra attention to the quantity and frequency of replenishment for the retailer, the parameters in the reorder point policy have significant influences on the inventory fluctuations for both retailer and manufacturer. Finally, to further quantify the production smoothing magnitude, the impact of all the decision parameters on the robust bullwhip metric is further studied based on a transfer function model. The results of this research provide insightful guidelines on the implementation of VMI program.

The remainder of this paper is organized as follows. Section 2 introduces related literature. Section 3 describes the VMI model in terms of the retailer subsystem and manufacturer subsystem. Section 4 studies the stability and inventory oscillations for the retailer subsystem. The production smoothing effect and inventory fluctuation for the manufacturer are studied in Section 5. Section 6 focuses on the robustness of the bullwhip effect problem under uncertain demand. This paper is concluded in Section 7.

\section{Literature Review}

Over the past decades, the advantages of VMI program have been extensively discussed. The shift of the authority of inventory control from downstream enables the supplier to choose the time and quantity for each replenishment order and also the route to transport the required goods to multiple retailers. It was well recognized that VMI brings more advantages over information sharing alone [4, 32-35]. In Fry et al. [14], a $(\mathrm{z}, \mathrm{Z})$ type contract was included in a VMI supply chain and the authors found that such a supply chain performs better than traditional supply chains. Lee et al. [3] studied the benefits and coordination problem for four business scenarios: traditional system, integrated system, VMI with stockout-cost sharing, and VMI with fixed transfer payments. Ben-Daya et al. [15] compared the benefits for three distinct supply chains, two of which were in the context of vendormanaged inventory consignment partnership. However, the supplier and retailer might belong to different companies or a vertically integrated company. The result showed that flexibility of the supplier has significant influences on the benefits of the vendor. Dong et al. [36] examined the benefits of the VMI program via empirical analysis, from which the results showed that inventory and stockout reductions might be realized at different times following the implementation of VMI. That is, inventory reduction initially may be the major benefit to distributor from VMI, while the benefits of stockout reduction may be realized after the first year. Lu et al. [9] studied the impact of two kinds of overconfidence on the inventory decision-making from a behavioral perspective. The study demonstrated behavioral factors might, to some extent, enhance the supplier's profit through continuous effort.

Although the opportunities of VMI are evident in many industries, the benefit allocation and coordination problem has been argued continuously [3, 37, 38]. A number of authors argued that the majority of the benefits from VMI tend to flow to the supplier, rather than to the retailer [32]. Lee and Cho [39] examined the problem of designing a VMI contract with consignment stock and stockout-cost sharing in a $(Q, r)$ inventory system between a supplier 
and a retailer. The result showed that the retailer may not always benefit from VMI. However, Mishra and Raghunathan [40] found that VMI intensifies the competition among manufacturers of competing brands, thus providing benefits to retailers. Pan and So [41] analyzed the interaction among the assembler and two component suppliers under a VMI contract. One supplier has uncertainty in the supply process, in which the actual number of components available for assembly is equal to a random fraction of the production quantity. Under deterministic and stochastic demand, the optimal component prices offered by the assembler are derived.

In addition to the previous literature, optimal replenishment policies of VMI supply chain systems with multiple retailers have been studied frequently over the recent years $[17,42-45]$. Nevertheless, we see that the existing literature has ignored the dynamic fluctuations of order and inventory, which can be hardly incorporated into the previous optimization or game-theory based models. Although our VMI model is similar to an early work by Disney and Towill [12], we generalized their model by allowing for free adjustment of the amount and timing of replenishment frequency. Another important difference is that we pay attention to the trade-off between the production smoothing and inventory fluctuations by providing some analytical results, while their work is focused on simulation experiments.

\section{Model Description and Analysis}

3.1. Description for the VMI System. Consider a periodicreview, single item VMI supply chain system composed of a retailer and a manufacturer. In a VMI program, the retailer should provide the manufacturer with demand and inventory information. On the basis of these information, the manufacturer makes replenishment decision to control the inventory for both the manufacturer and the retailer.

The timing of the events in each period is assumed as follows. At the beginning of each period $t$, the manufacturer puts its newly finished work-in-progress (WIP) inventory into a warehouse. Assume that there is a fixed production lead time, $L_{f}$, for the manufacturer to finish an order. At the same time, the retailer also receives its replenishment order from the manufacturer to recover its inventory. It takes the manufacturer $L_{r}$ periods to dispatch each replenishment order for the retailer. Note that one extra period is included in $L_{f}$ and $L_{r}$ to cope with the events order because the replenishment decisions are made at the beginning of each period. After putting final products into a warehouse, the manufacturer forecasts the demand for the coming period according to realized demand data. Then the manufacturer makes the production decision for itself and the replenishment decision for the retailer by reviewing the inventory information regarding the retailer's inventory level, in-transient inventory, and reorder point, as well as the manufacturer's own inventory level and WIP inventory. This is the essential difference in comparison with traditional supply chain models. To keep the model linear, we also assume that all the orders of the retailer which can not be satisfied immediately are backlogged. Finally, during the remaining time of each period, the retailer fulfils customer demand and any unfilled orders are also backlogged.

To be more general, we assume that the demand of the retailer is unknown but bounded by $D_{t} \in\left[d_{\min }, d_{\text {max }}\right]$, in which $d_{\min }$ and $d_{\text {max }}$ represent the lower bound and upper bound, respectively. The demand uncertainty is caused by a lot of factors, such as promotions, technological innovations, seasonal changes, and economical or political events. To cope with these uncertain factors, the manufacturer uses an adaptive reorder point policy to manage the retailer's inventory. The reorder point $R_{t}$ is updated by

$$
R_{t}=R_{t-1}+\theta_{r}\left(G \times D_{t-1}-R_{t-1}\right),
$$

where $\theta_{r}, 0 \leq \theta_{r} \leq 1$, is a coefficient to smooth the reorder point while the parameter $G$ is used to adjust service level. We note that the reorder point satisfies $G d_{\min } \leq$ $R_{t} \leq G d_{\text {max }}$ as long as $G d_{\text {min }} \leq R_{1} \leq G d_{\text {max }}$. Once the inventory position of the retailer, $I P_{r, t}$, is below the reorder point, an replenishment order will be triggered automatically or manually. The amount of the retailer's replenishment order $Q_{t}$ is determined by the following reorder point policy:

$$
Q_{t}= \begin{cases}\rho D_{t-1} & \text { if } I P_{r, t}<R_{t} \\ 0 & \text { if } I P_{r, t} \geq R_{t}\end{cases}
$$

in which the parameter $\rho$ is used to control the replenishment frequency for the retailer. Note that the reorder policy in Disney and Towill [12] is a special case when $\rho=1$, which imposes a constraint on the amount of each order and limits the decision-making flexibility for the manufacturer to adjust the replenishment frequency to achieve economical scale. In our model, we add this parameter $\rho$ allowing for freely adjusting the frequency of replenishment for the retailer, which essentially affects the performance of the whole system. Actually, the retailer might determine the value for $\rho$ according to its inventory capacity and demand characteristics. We argue that the parameter $\rho$ plays a different role in contrast to the parameter $G$ in (1). The parameter $\rho$ is used to determine the replenishment frequency, while $G$ is used to set the reorder point and balance the trade-off between inventory cost and service level. In practice, if the distance between the manufacturer and the retailer is large, the manufacturer might choose a large $\rho$ to save transportation cost. Finally, we advocate that the parameter setting for $G$ and $\rho$ should consider all the cost components.

In a VMI system, a vendor makes replenishment decisions based on information sharing with respect to downstream member's demand and inventory. In our periodic inventory model, the balanced equations for the retailer's inventory level, $I L_{r, t}$, and in-transient inventory, $G I T_{t}$, are, respectively, represented as

$$
I L_{r, t}=I L_{r, t-1}+Q_{t-L_{r}}-D_{t-1}
$$


and

$$
G I T_{t}=G I T_{t-1}-Q_{t-L_{r}}+Q_{t-1}
$$

The inventory position of the retailer is further obtained as $I P_{r, t}=I L_{r, t}+G I T_{t}$. Similarly, the difference equations for the manufacturer's inventory level and WIP are, respectively, represented as

$$
I L_{m, t}=I L_{m, t-1}+P_{t-L_{f}}-Q_{t-1}
$$

and

$$
W I P_{t}=W I P_{t-1}-P_{t-L_{r}}+P_{t-1}
$$

The exponential smoothing algorithm is accurate for short-term forecasting and easy to implement [30]; thus the manufacturer uses it to forecast the demand for the coming period. It should be noted that the change of reorder point should be incorporated into the forecasting process. The forecasting algorithm is obtained as

$$
F_{t}=F_{t-1}+\theta_{f}\left[D_{t-1}+\theta_{r}\left(G \times D_{t-1}-R_{t-1}\right)-F_{t-1}\right],
$$

where $\theta_{f}$ is the smoothing coefficient with $0 \leq \theta_{f} \leq 1$. A small $\theta_{f}$ helps smoothing order fluctuations, while a large $\theta_{f}$ is more appropriate to respond to volatile demand.

The replenishment rule used by the manufacturer for production decision is called APIOBPCS [30]. In traditional supply chains, the replenishment decisions for upstream members are made by themselves, whereas the replenishment decision in a VMI model should exploit systematic information. For this purpose, we define system inventory as

$$
S I_{t} \triangleq I L_{r, t}+I L_{m, t}+G I T_{t}-R_{t}
$$

The replenishment rule for the manufacturer is represented as

$$
P_{t}=F_{t}+\alpha^{i}\left(T I N T-S I_{t}\right)+\alpha^{w i p}\left(L_{m} \times F_{t}-W I P_{t}\right),
$$

where $\eta$ is the parameter to establish the target value of inventory level TINT $=\eta \times F(t), L_{m}$ is the production lead time, $L_{m} \times F_{t}$ is the desired value of WIP level, and $\alpha^{i}$ and $\alpha^{\text {wip }}$ are two replenishment parameters for recovering the inventory level and the WIP level. As the demand is uncertain, the target level for inventory and WIP should be updated adaptively. Essentially, the replenishment rule (9) is a proportional controller with two feedback loops. The two parameters significantly increases the flexibility to meet practical requirements. However, the feedback mechanism, the inventory interaction between the retailer and the manufacturer, coupled with the production lead time, makes the dynamics of VMI system highly complicated.

\subsection{A Transfer Function Model via Transformation of Differ-} ence Equations. In the literature, the production smoothing behavior is usually characterized by bullwhip effect metrics
[46]. To facilitate the study of production smoothing in terms of bullwhip effect, we develop a transfer function model by treating the demand of the retailer as input and the production of the manufacturer as output. Unlike existing methods in the literature, in which transfer functions are derived by combining the components of block graphs [30], we directly obtain the transfer function by formulating a state space model and manipulating the difference equations using z-transform [47]. This change avoids the complicated computation process of the well-known Mason's gain formula [48].

From the replenishment rule (9), we obtain

$$
\begin{aligned}
P_{t}-P_{t-1}= & {\left[1+\alpha^{i} \eta+\alpha^{w i p}\left(L_{f}-1\right)\right] \times\left(F_{t}-F_{t-1}\right) } \\
& -\alpha^{i}\left(S I_{t}-S I_{t-1}\right) \\
& -\alpha^{w i p}\left(W I P_{t}-W I P_{t-1}\right) .
\end{aligned}
$$

By the definition of system inventory, we can derive

$$
S I_{t}-S I_{t-1}=P_{t-L_{f}}-D_{t-1}-\theta_{r}\left(G D_{t-1}-R_{t-1}\right) .
$$

Substituting (6) and (11) into (10), we further have

$$
\begin{aligned}
P_{t} & -P_{t-1}=\left[1+\alpha^{i} \eta+\alpha^{\text {wip }}\left(L_{f}-1\right)\right] \\
\cdot & \theta_{f}\left[D_{t-1}+\theta_{r}\left(G \times D_{t-1}-R_{t-1}\right)-F_{t-1}\right] \\
& -\alpha^{i}\left[P_{t-L_{f}}-D_{t-1}-\theta_{r}\left(G D_{t-1}-R_{t-1}\right)\right] \\
& -\alpha^{w i p}\left(P_{t-1}-P_{t-L_{f}}\right) .
\end{aligned}
$$

From (12), it is very interesting to observe that for arbitrary demand processes, the parameter $\rho$ is irrelevant to the production dynamics. In traditional supply chains, order batching is frequently used to achieve transportation scale and reduce transportation cost. However, it is well recognized that order batching with low delivery frequency is one of the main causes of production fluctuations. In contrast to traditional supply chains, (12) demonstrates that, under the VMI program, the manufacturer can dispatch goods to the retailer with a low frequency to reduce transportation cost while not disrupting the execution of production schedules. In this sense, we conclude that transportation cost and production cost can be reduced simultaneously in a VMI system.

A state space model can be developed directly from (1), (7), and (12) by defining $X_{t} \triangleq\left(R_{t}, F_{t}, P_{t}\right)^{T}$ as the state vector, $U_{t} \triangleq D_{t}$ as input variable, and $Y_{t}$ as the output variable. Define $\psi\left(\alpha^{i}, \alpha^{w i p}, L_{f}\right) \triangleq 1+\alpha^{i} \eta+\alpha^{w i p}\left(L_{f}-1\right)$, a state space model is obtained as

$$
\begin{aligned}
X_{t} & =A X_{t-1}+A_{d} X_{t-L_{f}}+B U_{t} \\
Y_{t} & =C X_{t}
\end{aligned}
$$

where 


$$
\begin{aligned}
A & =\left[\begin{array}{ccc}
1-\theta_{r} & 0 & 0 \\
-\theta_{f} \theta_{r} & 1-\theta_{f} & 0 \\
-\alpha^{i} \theta_{r}-\theta_{r} \theta_{f} \times \psi\left(\alpha^{i}, \alpha^{w i p}, L_{f}\right) & -\theta_{f} \times \psi\left(\alpha^{i}, \alpha^{w i p}, L_{f}\right) & 1-\alpha^{w i p}
\end{array}\right], \\
A_{d} & =\left[\begin{array}{ccc}
0 & 0 & 0 \\
0 & 0 & 0 \\
0 & 0 & \alpha^{w i p}-\alpha^{i}
\end{array}\right], \\
B & =\left[\begin{array}{c}
G \theta_{r} \\
\theta_{f}\left(1+\theta_{r} G\right) \\
\theta_{f}\left(1+\theta_{r} G\right) \times \psi\left(\alpha^{i}, \alpha^{w i p}, L_{f}\right)+\alpha^{i}\left(1+\theta_{r} G\right)
\end{array}\right],
\end{aligned}
$$

and

$$
C=\left[\begin{array}{lll}
0 & 0 & 1
\end{array}\right] .
$$

Using the z-transform in Jury [47], the state space model (13) is converted as

$$
X(z)=\left(I-A z^{-1}-A_{d} z^{-L_{f}}\right) B z^{-1} U(z) .
$$

Finally, we can obtain the following transfer function:

$$
\begin{aligned}
G(z) & =\frac{P(z)}{D(z)}=\frac{Y(z)}{U(z)} \\
& =C\left(I-A z^{-1}-A_{d} z^{-L_{f}}\right)^{-1} B z^{-1} .
\end{aligned}
$$

As mentioned in Lalwani [49], state space model (13) lays a solid foundation for studying the controllability and observability problem, and it might open a door for studying nonlinear dynamics and incorporating time-varying lead time into the APIOBPCS model, which makes the model more realistic and the results appealing. In contrast to the existing literature, the transfer function (17) in this study is formulated in a matrix form, which facilitates the implementation in computers. More importantly, the derivation process is simpler than obtaining the transfer function by combining block graphs with Mason's gain formula [48], which enables us study the production smoothing behavior in terms of worst-case bullwhip for unknown demand.

\section{Inventory Fluctuations of the Retailer}

Before we discuss the dynamics of the VMI system, we shall firstly introduce the definition of stability. By stability, we mean that the output of a system is bounded if the input is bounded. The customer demand $D_{t}$ is treated as the input. Because the fluctuations of inventory and order are of great importance, we define that the retailer subsystem is stabilized if $I L_{r, t}$, or $I P_{r, t}$, is bounded. Note that $Q_{t}$ is easily bounded because customer demand is bounded by nature. The manufacturer subsystem is stable if $I L_{m, t}$ and $P_{t}$ are bounded.
Although the stability of the VMI system has been discussed in Disney and Towill [30], the obtained results are limited to small or specified lead times. Furthermore, the previous literature has ignored the interaction between the retailer and manufacturer. In particular, they failed to consider the dynamics of the retailer, which might be due to the fact that the model in Disney and Towill [30] only considers the special case $\rho=1$, which simplifies the dynamics of the retailer subsystem.

In this section, we begin by studying the stability and inventory oscillation of the retailer. In the VMI program, the retailer may assume the risk of incurring high inventory cost or poor customer service level. To avoid such a problem, the retailer can increase its benefits by signing effective contractual agreements, which are characterized by the penalty cost once the retailer's inventory beyond an upper bound or a lower bound. To reduce the penalty cost and maintaining satisfactory service level for the retailer, the manufacturer must clarify how the replenishment rule affects the range of inventory fluctuations of the retailer. To this end, as a first step, we shall now discuss the inventory fluctuation range of the retailer under uncertain demand with Theorem 1.

Theorem 1. Assume that $R_{1}<I_{r} \leq(G+\rho) d_{\max }-d_{\min }$ and $\rho \geq 1$. Define $I L_{r, t}^{\prime}=I L_{r, t}-D_{t}$ as the inventory level of the retailer at the end of each period, then the retailer's inventory position satisfies

$$
G d_{\text {min }}-d_{\text {max }} \leq I P_{r, t} \leq(G+\rho) d_{\text {max }}-d_{\text {min }} ;
$$

and $I L_{r, t}^{\prime}$ satisfies

$$
\begin{aligned}
& G d_{\text {min }}-\left(L_{r} \rho+2\right) d_{\text {max }} \leq I L_{r, t}^{\prime} \\
& \leq(G+\rho) d_{\text {max }}-2 d_{\text {min }} .
\end{aligned}
$$

Proof. Let $I_{r}$ be the initial inventory of the retailer and $R_{1}$ be the reorder point in the first period. We set $I_{r}>R_{1}$ and $G d_{\text {min }} \leq R_{1} \leq G d_{\text {max }}$. Because the inventory position is less than the reorder point, the manufacturer will not dispatch goods to the retailer until the retailer's inventory position drops below the reorder point. The dynamics for the retailer's 
inventory level and reorder point are depicted in Figure 1. Without loss of generality, we assume that $I P_{r, t} \geq R_{t}$ for $t<t_{1}^{*}$, $I P_{r, t}<R_{t}$ for $t_{1}^{*} \leq t<t_{2}^{*}$, and $I P_{r, t} \geq R_{t}$ when $t=t_{2}^{*}$. In the first stage $t<t_{1}^{*}$, we have $Q_{t}=0$ and $G d_{\text {min }} \leq R_{t} \leq I P_{r, t}$. In the second stage $t_{1}^{*} \leq t<t_{2}^{*}$, because $I P_{r, t}<R_{t}$ the manufacturer should send the replenishment order to the retailer, which is given by $Q_{t}=\rho D_{t-1}$. For $t=t_{1}^{*}$, we have

$$
I P_{r, t_{1}^{*}}=I P_{r, t_{1}^{*}-1}-D_{t_{1}^{*}-1} \geq G d_{\min }-d_{\max }
$$

When $t_{1}^{*}<t<t_{2}^{*}$

$$
I P_{r, t_{1}^{*}+1}=I P_{r, t_{1}^{*}-1}-D_{t_{1}^{*}-1}+\rho D_{t_{1}^{*}-1}-D_{t_{1}^{*}}
$$

and

$$
I P_{r, t}=I P_{r, t_{1}^{*}-1}+\sum_{i=0}^{t-1-t_{1}^{*}}(\rho-1) D_{t_{1}^{*}-1+i}-D_{t-1}
$$

Because $\rho \geq 1$, the upper bound and lower bound for the inventory position $I P_{r, t}$ are obtained by

$$
G d_{\min }-d_{\max } \leq I P_{r, t} \leq G d_{\max }
$$

Inequality (23) implies that there exists a lower bound for $I P_{r, t}$ once it drops below the reorder point. In the third stage $t \geq t_{2}^{*}$, we still have $G d_{\text {min }} \leq R_{t} \leq I P_{r, t}$ and

$$
\begin{aligned}
I P_{r, t_{2}^{*}} & =I P_{r, t_{2}^{*}-1}+\rho D_{t_{2}^{*}-2}-D_{t_{2}^{*}-1} \\
& \leq(G+\rho) d_{\text {max }}-d_{\text {min }} .
\end{aligned}
$$

Further, we can obtain

$$
I P_{r, t_{2}^{*}+1}=I P_{r, t_{2}^{*}-1}+\rho D_{t_{2}^{*}-2}-D_{t_{2}^{*}-2}-D_{t_{2}^{*}-1} \leq I P_{r, t_{2}^{*}}
$$

It means that once the inventory position is higher than the reorder point, it starts to decrease. The inventory level at the end of each period for the retailer is represented as

$$
I L_{r, t}^{\prime}=I P_{r, t}-\sum_{i=1}^{L_{r}} Q_{t-i}-D_{t}
$$

Further, we directly obtain

$$
\begin{aligned}
& G d_{\text {min }}-\left(L_{r} \rho+2\right) d_{\text {max }} \leq I L_{r, t}^{\prime}=I P_{r, t}-\sum_{i=1}^{L_{r}} Q_{t-i}-D_{t} \\
& \quad \leq(G+\rho) d_{\text {max }}-2 d_{\text {min }} .
\end{aligned}
$$

It completes the proof of Theorem 1.

From Theorem 1, we see that the retailer subsystem is BIBO stable if and only if $\rho \geq 1$. When $\rho<1$, the retailer subsystem will be destabilized because its demand can not be sufficiently satisfied and its inventory will decrease continuously. In addition, Theorem 1 also provides us some managerial insights. The retailer's service level is mainly determined by the parameter $G$, which is employed to set the reorder point. A large $G$ results in better customer service

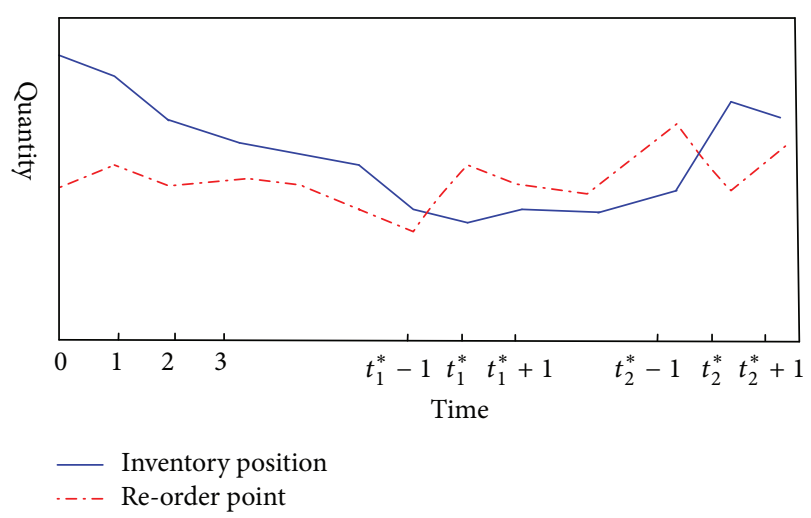

FIGURE 1: The schematic dynamic curves for the retailer's inventory position and reorder point.

level but incurs high inventory cost and leads to negative effect to cause huge inventory oscillations. As mentioned, the delivery frequency for the retailer is determined by $\rho$; that is, a large value of $\rho$ can be exploited to reduce transportation cost. However, high delivery frequency also increases inventory fluctuations. As the replenishment lead time increases, the retailer's inventory cost might increase. Actually, it is well recognized that lead time is one of the main causes of the bullwhip effect. Finally, demand uncertainties are also key drivers for high inventory cost.

\section{Inventory and Production Fluctuations of the Manufacturer}

Under the VMI program, the manufacturer manages both its own inventory and the retailer's inventory based on the systematic information. The shifting of the authority of inventory control and information sharing, two main properties of a VMI supply chain system, must affect the dynamics properties of the entire system. Disney and Towill [30] studied the stability of a similar VMI supply chain model using transfer function approach. However, they only provided the stability results for small lead times while ignoring inventory fluctuations of the VMI supply chain system. For example, the shipment policy used by the manufacturer to control the retailer's inventory will certainly affect the retailer's dynamics, as emphasized in the previous section. Meanwhile, the retailer's dynamics have great influences on the dynamics of the manufacturer. Wei et al. [21] studied the stability of the discrete APIOBPCS model for a single manufacturer, the methods in which can be extended to a two-echelon VMI supply chain system in Section 4.

As mentioned, if $0<\theta_{r}<1$ and $0<\theta_{f}<1$, the two variables $F_{t}$ and $R_{t}$ will be bounded within certain ranges. Because final demand and demand forecast signal can be treated as external perturbations, from (12), we see that the stability of the production process of the manufacturer is determined by

$$
P_{t}=\left(1-\alpha^{w i p}\right) P_{t-1}+\left(\alpha^{w i p}-\alpha^{i}\right) P_{t-L_{f}}
$$


We recall that $\rho<1$ will result in more and more inventory shortages for the retailer. In this situation, the retailer subsystem can be hardly stable. Then, an interesting phenomenon arises. The manufacturer's production process can be stable even if the retailer's inventory is unstable. Here, we must distinguish the stability of the manufacturer's production process from the stability of the manufacturer subsystem. We say that the production process is stable as long as the variable $P_{t}$ is bounded, while the stability for the manufacturer subsystems requires both its inventory related variables, such as $S I_{t}$ and $I L_{m, f}$, and production process $P_{t}$ are all bounded. Actually, this interesting result is due to the fact that the production decision of the manufacturer is made based on system inventory $S I_{t}$, which is defined as $S I_{t}=$ $I P_{r, t}-R_{t}+I L_{m, f}$, rather than $I L_{m, f}$. Thus, we can get the conclusion that even though $\rho<1$, the production process can be stable, but the manufacturer's inventory will increase continuously. Thus, we can draw an initial conclusion that there must be a trade-off between inventory fluctuations and production fluctuations. This result is also the key difference between VMI systems and traditional supply chain systems. This result will be further discussed with more details by simulation experiments later. As a result, if $\rho \geq 1$, the stability of (28) guarantees the stability for the entire VMI supply chain system.

In fact, the stability of (28) has been discussed in Wei et al. [21] by studying a single manufacturer. Here we can obtain a necessary and sufficient stability condition for the VMI supply chain by Theorem 2 .

Theorem 2. Let $L_{f} \geq 2$. Then the VMI supply chain is stable if and only if $\left|1-\alpha^{\text {wip }}\right|<L_{f} /\left(L_{f}-1\right), \rho \geq 1$, and

(a) $\left|1-\alpha^{\text {wip }}\right|-1<\alpha^{i}-\alpha^{\text {wip }}<\left[2+\left(\alpha^{\text {wip }}\right)^{2}-2 \alpha^{\text {wip }}-2 \mid 1-\right.$ $\left.\alpha^{\text {wip }} \mid \cos \phi\right]^{1 / 2}$ for even $L_{f}$, or

(b) $\left|1-\alpha^{i}\right|<1$ and $\left|\alpha^{i}-\alpha^{w i p}\right|<\left[2+\left(\alpha^{\text {wip }}\right)^{2}-2 \alpha^{\text {wip }}-\right.$ $\left.2\left|1-\alpha^{w i p}\right| \cos \phi\right]^{1 / 2}$ for odd $L_{f}$,

where $\phi$ is the solution in $\left(0, \pi / L_{f}\right)$ of $\left|1-\alpha^{\text {wip }}\right|=$ $\sin \left(L_{f} \phi\right) / \sin \left(L_{f} \phi-\phi\right)$.

It should be remarked that $\left|1-\alpha^{\text {wip }}\right|<L_{f} /\left(L_{f}-1\right)$ guarantees only one solution for $\left|1-\alpha^{w i p}\right|=\sin \left(L_{f} \phi\right) / \sin \left(L_{f} \phi-\right.$ $\phi)$ in $\left(0, \pi / L_{f}\right)$. From Theorem 2 , we see that the stability boundaries for even or odd production lead times are quite different. Based on Theorem 2, we can draw precise stability boundaries or stable regions for arbitrary production lead times.

In order to simplify the selection of replenishment parameters ensuring the system is stable, we can further obtain the stability condition independent of the production lead time, which is introduced in Theorem 3. The proof for Theorem 3 is similar to the proof for the Proposition 4.3 in Wei et al. [21] and we will not include the details here.

Theorem 3. The VMI supply chain is stable if $\rho \geq 1$ and

$$
\left|1-\alpha^{w i p}\right|+\left|\alpha^{w i p}-\alpha^{i}\right|<1 .
$$

In fact, the precise stable regions gradually converge to the stable region independent of production lead times as the production lead time increases. Figure 2 shows the stable regions in the $\alpha^{\text {wip }}-\alpha^{i}$ plane for different production lead times, in which $P$ - Region represents precise stable regions tailored for specific lead times with Theorem 2, and S-Region represents the stable region independent of lead times. It is obvious that the area of $S$-Region will cover most of the areas of the precise stable region $P$ - Region as the production lead time increases. It implies that the $S$ - Region simplifies the selection of replenishment parameters.

From above, we see that the production process of the manufacturer can be smoothed even with low delivery frequency determined by the manufacturer to control retailer's inventory. In this sense, the manufacturer can reduce transportation cost and production cost simultaneously. However, the manufacturer must be cautious to determine the replenishment frequency or the size of order batching, because there still exists a trade-off between inventory cost and production cost caused by production fluctuations. This trade-off will be further explored via simulation experiments in the next section.

\section{Simulation Experiments}

In the simulation experiments, we will firstly validate the theoretical results on the range of inventory fluctuations of the retailer subsystem. Then, we will study the dynamics interaction between the retailer and the manufacturer. Finally, the impact of different parameters on the production smoothing will be numerically studied with the worst-case bullwhip measure.

6.1. Inventory Fluctuations of the Retailer. We will validate the theoretical results with uniformly distributed demand $D_{t} \sim$ $\left[d_{\text {min }}, d_{\text {max }}\right]$. The reason we choose uniformly distributed demand is that the upper bound and lower bound can be readily known. It is worth noting that the above results can be applied to other demand functions as long as we know the maximal value and the minimal value of the demand data. Based on Theorem 1 , we define $\wp_{1} \triangleq(G+\rho) d_{\text {max }}-2 d_{\text {min }}$ and $\wp_{2} \triangleq G d_{\text {min }}-\left(L_{r} \rho+2\right) d_{\text {max }}$ as the upper bound and lower bound of the retailer's inventory, respectively. Further, we measure the range of inventory fluctuation as

$$
\begin{aligned}
\Delta_{\wp} & \triangleq \wp_{1}-\wp_{2} \\
& =(G+2)\left(d_{\text {max }}-d_{\text {min }}\right)+\rho\left(1+L_{r}\right) d_{\text {max }} .
\end{aligned}
$$

In the simulations, we compute the retailer's system performance by four metrics: replenishment frequency, $F R_{r}$, the customer service level metric, $S L_{r}$, the maximal value of inventory level, $I L_{\max }$, and the minimal value of inventory level, $I L_{\text {min }}$. Assume that we simulate the retailer's dynamics for $N$ periods. Then the metric $F R_{r}$ is represented by

$$
F R_{r} \triangleq \frac{C\left(Q_{t}\right)}{N},
$$

where $C\left(Q_{t}\right)$ records the number of periods that the manufacturer should deliver a replenishment order to the retailer 


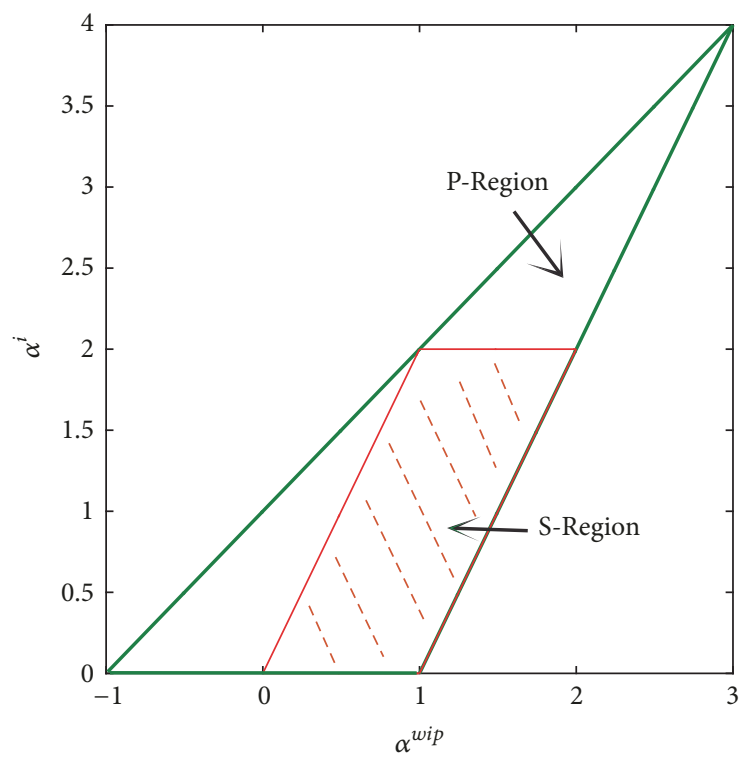

(a) $L_{f}=2$

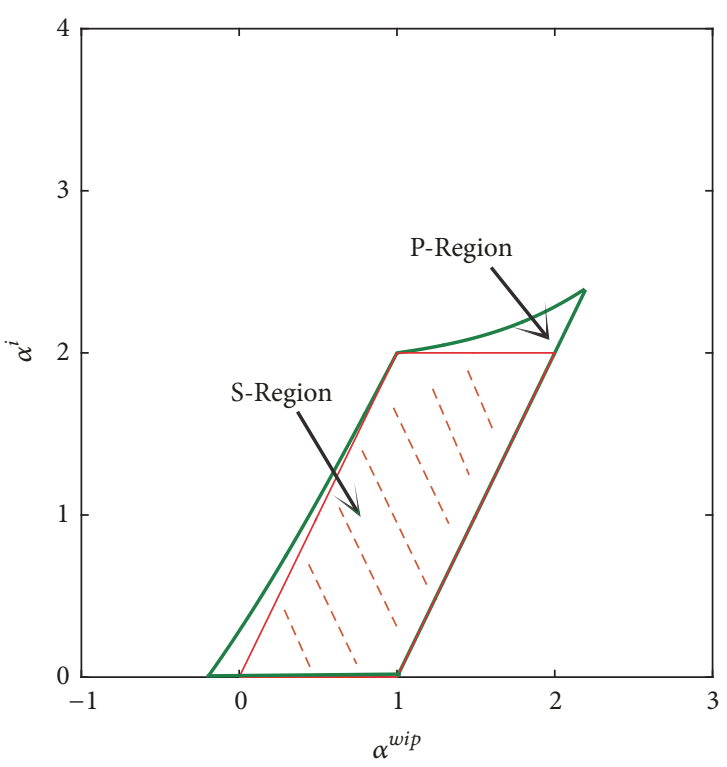

(c) $L_{f}=6$

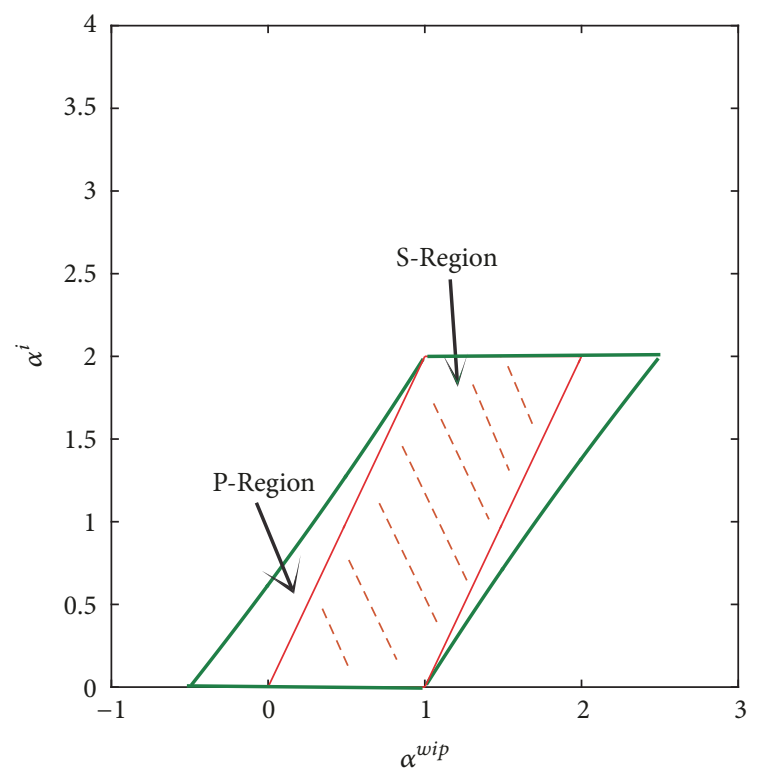

(b) $L_{f}=3$

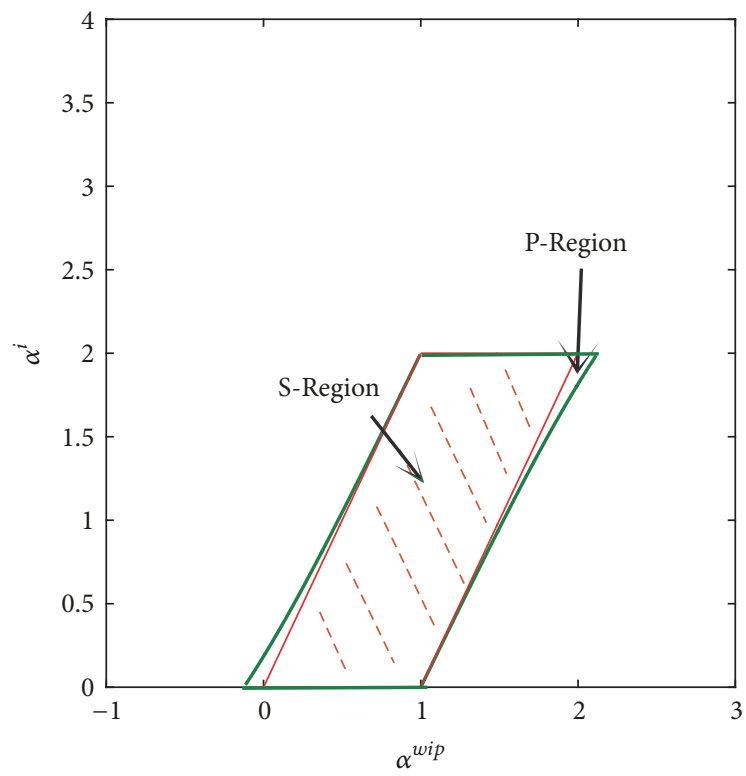

(d) $L_{f}=9$

FIGURE 2: The stability boundaries for the VMI supply chain system.

$\left(Q_{t}>0\right)$. It means that a higher $F R_{r}$ will result in a higher frequency of replenishment order. As a result, the metric $F R_{r}$ is closely relevant to the transportation cost. The transportation cost can be saved for a small $F R_{r}$. The customer service level metric $S L_{r}$ is represented as

$$
S L_{r}=\frac{S\left(I L_{r, t}^{\prime}\right)}{N}
$$

where $S\left(I L_{r, t}^{\prime}\right)$ is the number of periods satisfying $I L_{r, t}^{\prime}>0$. The service level is high if the value of $S L_{r}$ is large.

The other two metrics $I L_{\max }$ and $I L_{\min }$ are defined as

$$
I L_{\text {max }}=\max \left\{I L_{N_{0}}^{\prime}, I L^{\prime}, \ldots, I L_{N}^{\prime}\right\}
$$

and

$$
I L_{\text {min }}=\max \left\{I L_{N_{0}}^{\prime}, I L^{\prime}, \ldots, I L_{N}^{\prime}\right\},
$$

in which $N_{0}$ is the starting period used to eliminate the influence of initial data on system performance. Based on these four metrics, four groups of simulations are designed to observe the impact of demand fluctuations, $G, \rho$, and $L_{r}$ on system performance. In the simulation, we set $N=5000$, $N_{0}=200$, and $I_{r}=100$. Theoretical results and simulation results are compared in Table 1.

Table 1 well validates the results obtained previously. The first group of simulation experiment $G_{1}$ demonstrates that demand fluctuations are closely related to inventory 
TABLE 1: The comparison between simulation results and theoretical results for the retailer subsystem.

\begin{tabular}{ccccccccccccc}
\hline \multirow{3}{*}{ Groups } & \multicolumn{4}{c}{ Parameter values } & \multicolumn{4}{c}{ Theoretical results } & \multicolumn{4}{c}{ Simulation results } \\
& $d_{\min }$ & $d_{\max }$ & $G$ & $\rho$ & $L_{r}$ & $\wp_{1}$ & $\wp_{2}$ & $\Delta_{\wp}$ & $F R_{r}$ & $S L_{r}$ & $I L_{\max }$ & $I L_{\min }$ \\
\hline \multirow{3}{*}{$G_{1}$} & $\mathbf{1 0}$ & $\mathbf{4 0}$ & 4 & 1.5 & 2 & -160 & 200 & 360 & 0.57 & 0.99 & 128.30 & -27.11 \\
& $\mathbf{1 0}$ & $\mathbf{8 0}$ & 4 & 1.5 & 2 & -360 & 420 & 780 & 0.52 & 0.97 & 265.67 & -63.48 \\
& $\mathbf{1 0}$ & $\mathbf{1 2 0}$ & 4 & 1.5 & 2 & -560 & 640 & 1200 & 0.50 & 0.95 & 372.84 & -125.67 \\
\hline \multirow{3}{*}{$G_{2}$} & 10 & 40 & $\mathbf{2}$ & 1.5 & 2 & -180 & 120 & 300 & 0.58 & 0.43 & -60.96 & 64.32 \\
& 10 & 40 & $\mathbf{6}$ & 1.5 & 2 & -100 & 260 & 360 & 0.52 & 1.00 & 185.89 & 7.28 \\
& 10 & 40 & $\mathbf{1 0}$ & 1.5 & 2 & -180 & 300 & 480 & 0.54 & 1.00 & 298.74 & 98.37 \\
\hline \multirow{3}{*}{$G_{3}$} & 10 & 40 & 6 & $\mathbf{1}$ & 2 & -100 & 260 & 360 & 1.00 & 0.48 & 44.30 & -40.93 \\
& 10 & 40 & 6 & $\mathbf{2}$ & 2 & -180 & 300 & 480 & 0.41 & 1.00 & 199.87 & 19.29 \\
& 10 & 40 & 6 & $\mathbf{5}$ & 2 & -420 & 420 & 840 & 0.16 & 1.00 & 307.03 & 17.53 \\
\hline \multirow{3}{*}{$G_{4}$} & 10 & 40 & 4 & 1.5 & $\mathbf{3}$ & -220 & 200 & 420 & 0.43 & 0.81 & 101.57 & -53.32 \\
& 10 & 40 & 4 & 1.5 & $\mathbf{5}$ & -340 & 200 & 540 & 0.41 & 0.16 & 72.70 & -108.38 \\
& 10 & 40 & 4 & 1.5 & $\mathbf{7}$ & -460 & 200 & 660 & 0.565 & 0.01 & 22.90 & -173.32 \\
\hline
\end{tabular}

fluctuations. Meanwhile, demand uncertainty could result into poor customer service level. The second group $G_{2}$ shows that increasing the parameter $G$ will improve customer service level. However, high inventory fluctuations will occur if the parameter $G$ is sufficiently large. In the third group of simulation, it is obvious that increasing $\rho$ increases the delivery frequency for the retailer. This means that the generalized replenishment policy (2) is more flexible in determining the replenishment frequency than $\rho=1$. However, Theorem 1 illustrates that a large $\rho$ contributes to inventory fluctuations. Actually, in the third group of simulation $G_{3}$, it is also interesting to observe that $\rho=1$ can make the manufacturer lose control of the retailer's inventory because $F R_{r}=1$. This situation leads to low customer service level because the reorder point loses its ability to limit the retailer's inventory. The final simulation group $G_{4}$ validates the result that long lead time brings difficulties for inventory control.

6.2. Production and Inventory Fluctuations of the Manufacturer. We shall now simulate the system with a unit step signal as the demand input, which is represented by

$$
D_{t}= \begin{cases}1 & \text { if } t \geq 1 \\ 0 & \text { if } t<1\end{cases}
$$

The step signal is widely used in testing dynamical systems. Actually, the step signal can be used to model sudden change of customer demand due to accidental events. The wellknown Supply Chain Operations Reference (SCOR) model also measures supply chain performance in response to a step change in demand. In this subsection, we select a common group of parameter setting: $\theta_{r}=\theta_{f}=1 / 9, G=4, L_{r}=2$, $I_{r}=5, R_{1}=0$, and $I_{f}=0$. In the following, in order to figure out the reason why the production process can be stabilized even if the retailer subsystem is destabilized with $\rho<1$, we will firstly explore the dynamic properties under two different scenarios, namely, $\rho<1$ and $\rho>1$. Then, we will validate the stability results derived previously.
6.2.1. Production and Inventory Dynamics. In the case when $\rho<1$, the retailer subsystem is destabilized. In the simulation experiments, we firstly set $\rho=0.8, \alpha^{i}=0.25$, and $\alpha^{\text {wip }}=0.5$. The production and inventory dynamics for the VMI system in response to the step signal input are shown in Figures 3(a) and 3(b). Figure 3(a) shows that the production process, $P_{t}$, and system inventory, $S I_{t}$, are stable. However, $\rho<1$ causes serious outcome that the inventory levels for both the retailer and the manufacturer are divergent or unstable. In particular, the customer demand of the retailer can never be fully satisfied because the replenishment amount is always less than realized demand. Even if the production process is smoothed, the manufacturer accumulates high inventory, which will further lead to extra inventory cost. Thus, we should avoid selecting the parameter setting $\rho<1$.

The advantages of $\rho>1$ are shown in Figures 3(c) and $3(\mathrm{~d})$ by setting $\rho=3, \alpha^{i}=0.25$, and $\alpha^{\text {wip }}=0.5$. This parameter setting falls into the stable region determined by Theorems 2 and 3. In this situation, the delivery frequency is decreased to reduce transportation cost. Although the replenishment order for the retailer fluctuates with a large magnitude, the production dynamics is still smoothed. In this sense, the production cost due to machine startup or labor force scheduling can be saved. However, the manufacturer should be cautious on selecting the delivery frequency or determining the value for $\rho$, as the inventory fluctuations magnitude might be large for both the retailer and the manufacturer, which increases inventory cost for the retailer and penalty cost for the manufacturer. As introduced in the previous section, $\rho=1$ may lead to a situation that the reorder point makes no sense for large value of $G$, while incorporating the parameter $\rho$ improves the flexibility for the manufacturer to manage the retailer's inventory greatly. In practice, managers should make a balanced decision towards transportation cost, inventory cost, and production cost.

6.2.2. Validation for Stability Results. Here we simply test the stability results for $\rho=1, L_{f}=9$, and $\alpha^{\text {wip }}=1.2$. In such 

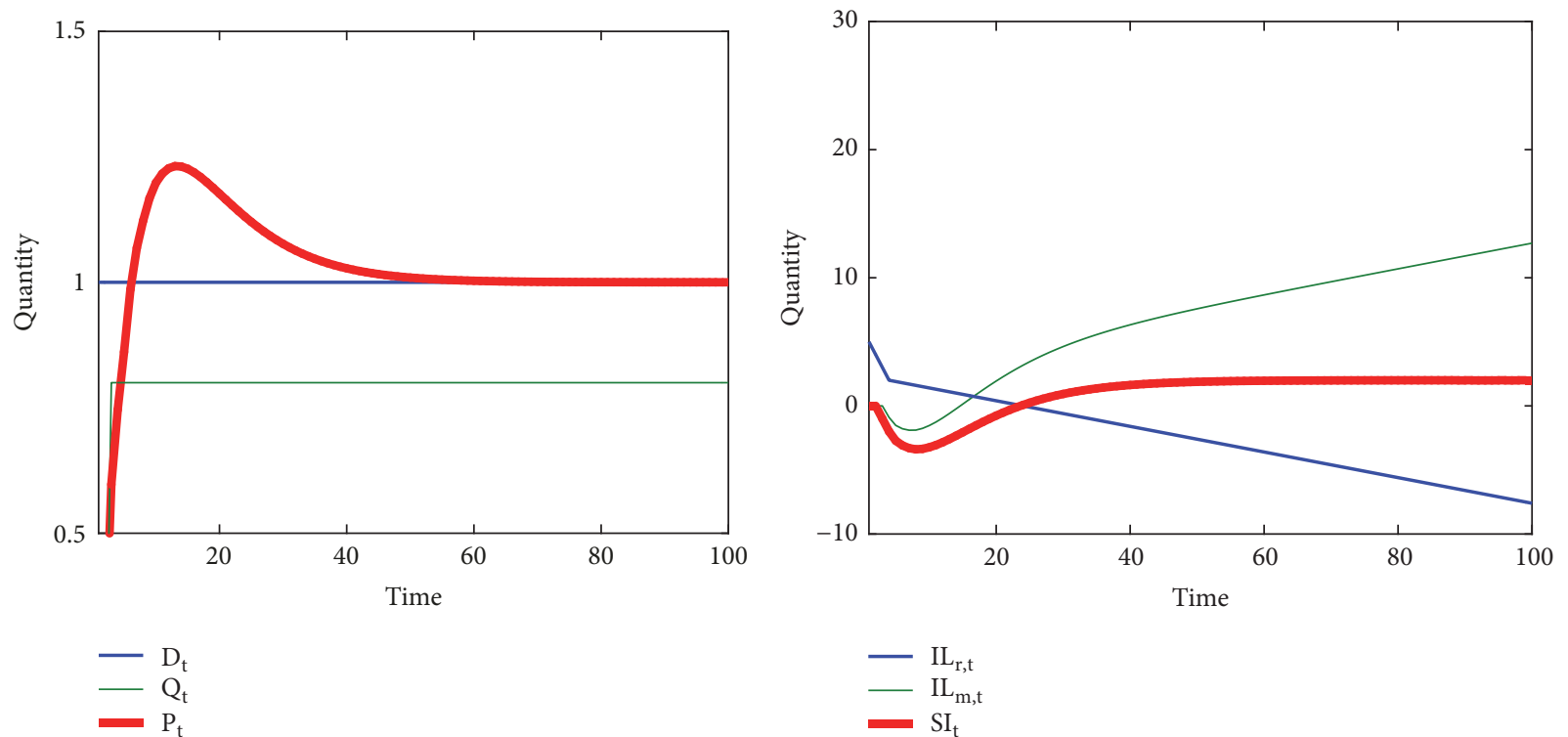

(a) Production and order dynamics when $\rho<1$

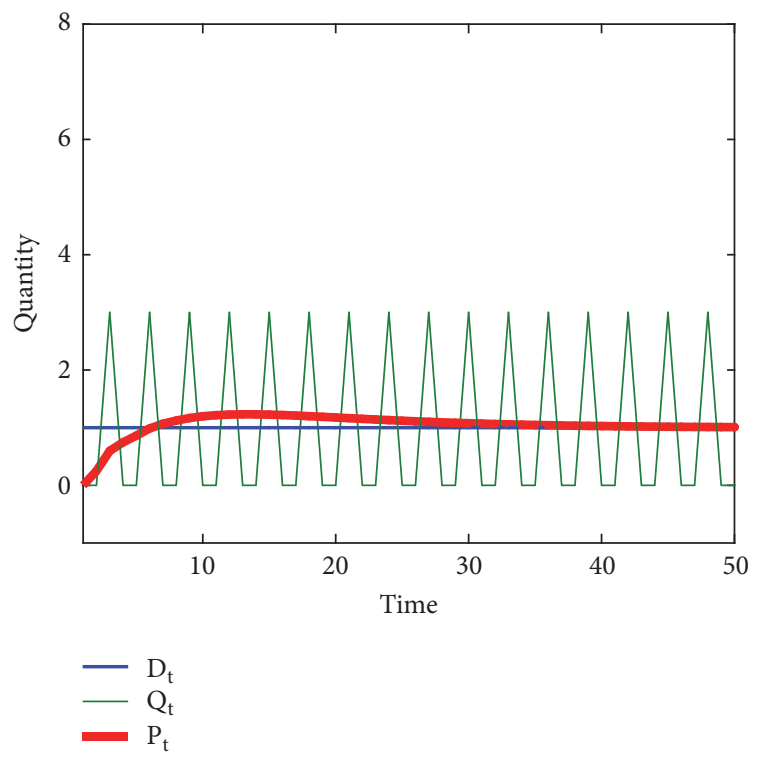

(b) Inventory dynamics when $\rho<1$



(c) Production and order dynamics when $\rho>1$

(d) Inventory dynamics when $\rho>1$

FIgURE 3: The inventory and production dynamics for the VMI supply chain system.

a situation, the necessary and sufficient stable condition for the VMI supply chain system becomes $0.3858<\alpha^{i}<2$. In the simulation, we consider two scenarios: unstable scenario, $\alpha^{i}=0.32$, and stable scenario, $\alpha^{i}=0.42$. The inventory dynamics for the VMI supply chain system are shown in Figure 4. Figure 4(a) shows the dynamics for $\alpha^{i}=0.32$, in which all the state variables diverge as time passes. Unstable design always bring high inventory and production cost as a result of inventory fluctuations. Thus, unstable behavior should be avoided by selecting appropriate replenishment parameters. In contrast, Figure 4(b) shows the stable behavior for $\alpha^{i}=0.42$. In a stable system, inventory and production will return to steady state even under sudden change of customer demand.
6.3. A Worst-Case Bullwhip Effect Study. For a manufacturer, reducing production fluctuations is significant because it is closely related to production cost. Smoothing production process helps arranging labor force, deciding the manufacturing capacity, and also enlarging the life of production machines. It is well recognized that production fluctuations can be smoothed by mitigating the bullwhip effect [46]. Actually, stability is a fundamental step for smoothing production fluctuations because it makes no sense to study the bullwhip for an unstable supply chain system. In the following, based on the stability conditions in Theorems 2 and 3, we will further study the production smoothing behavior in terms of the worst-case bullwhip effect based on the transfer function model (17). 


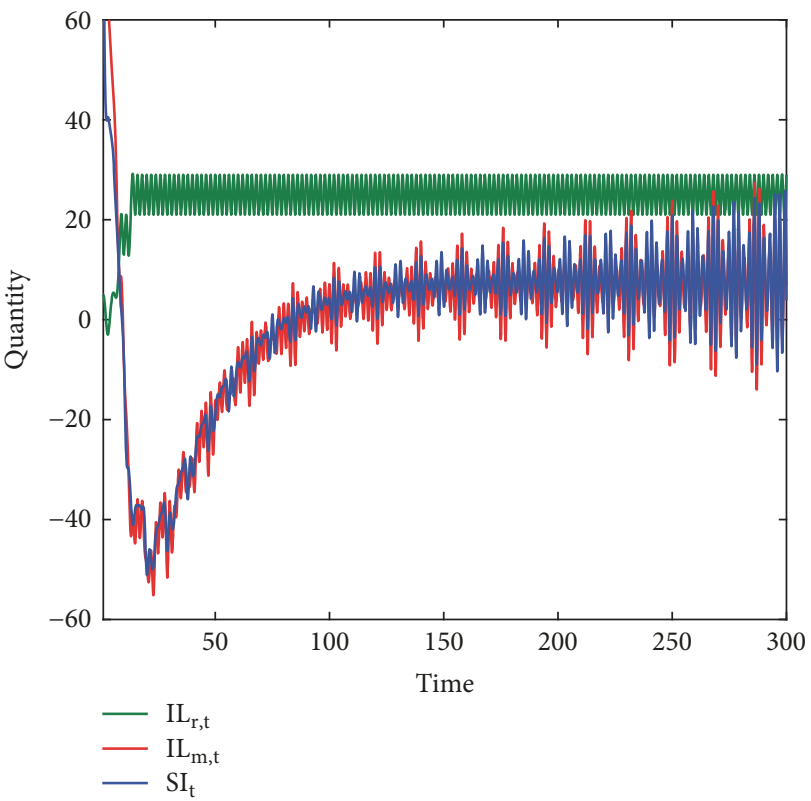

(a) $\alpha^{i}=0.32, \alpha^{\text {win }}=1.2$

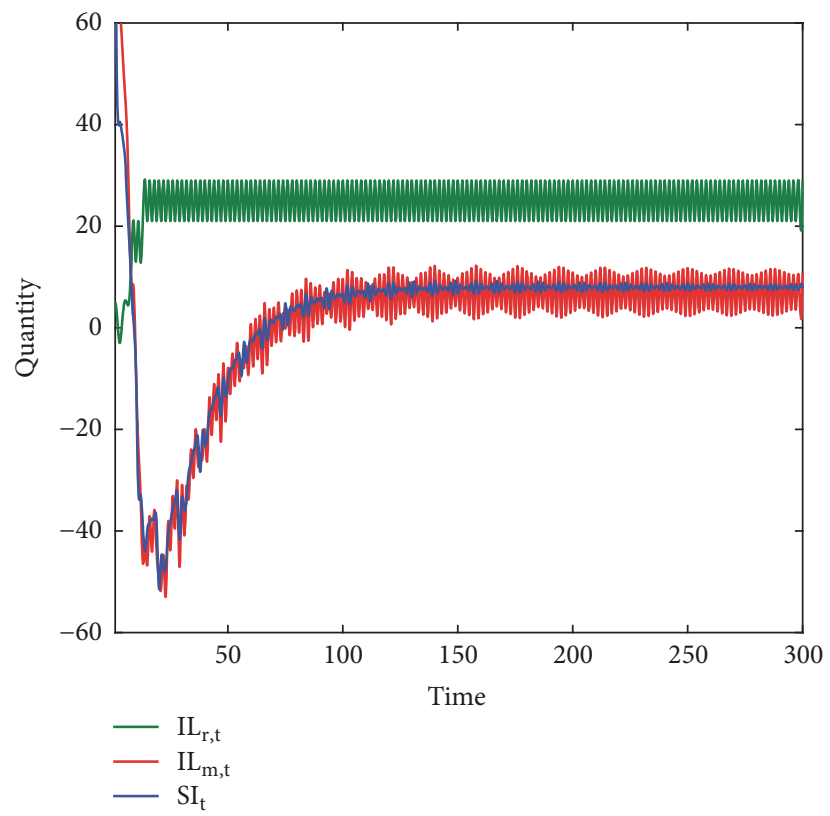

(b) $\alpha^{i}=0.42, \alpha^{\text {win }}=1.2$

FIGURE 4: Validation of stability results.

The bullwhip effect problem has received considerable attention since it causes high cost for upstream firms in a supply chain firm due to the distortion of demand information. The majority of the literature focuses on the bullwhip effect of supply chain system for specific demand with statistical method and control theory method [50]. However, the customer demand in real life is highly uncertain over time and the probability distribution used to approximate real life demand can be inaccurate or unreliable [51]. To this end, we aim to understand the bullwhip effect of the VMI supply chain by combining the state space model and transfer approach for unknown demand. As mentioned, the state space model can be readily constructed from the difference model, which can be conveniently used to derive transfer functions. As mentioned, it is complicated to derive transfer functions by combining block graphs. Here we study the bullwhip effect for unknown demand using transfer function because it is easier to calculate the robust bullwhip metric.

Assume that $G(z)$ is the transfer function by selecting $P_{t}$ as output and $D_{t}$ as input in the frequency domain. Then the worst-case bullwhip effect metric $[21,52]$ is represented as

$$
W_{I}^{2}:=\left(\sup _{w \in[0,2 \pi)}|G(z)|_{z=e^{j w}}\right)^{2}
$$

where $|G(z)|$ is the modulus of the transfer function $G(z)$. From the definition (36), we see that the metric $W_{I}^{2}$ is the squared worst-case amplification of $G(z)$ across all $w \in$ $[0,2 \pi)$. The most important property is that $W_{I}^{2}$ is independent of customer demand. In particular, the VMI supply chain system experiences no bullwhip effect when $W_{I}^{2}<1$.
Using Matlab 7.0, we can calculate the value of $W_{I}^{2}$ based on the transfer function model (17) accurately. The following numerical analysis will disclose the influences of replenishment parameters, production lead time, and forecasting parameters on the worst-case bullwhip effect for unknown demand. We see that the parameter $\rho$ is not included in the state space model (13) and thus it has no influence on the smoothness of production fluctuations. Meanwhile, the robust metric $W_{I}^{2}$ is irrelevant to the retailer's lead time $L_{r}$. Here we focus on the influences of $\alpha^{i}, \alpha^{w i p}, G$, and $\theta_{f}$ on the worst-case bullwhip effect.

When $L_{f}=3, \eta=1, G=4$, and $\theta_{r}=\theta_{f}=$ 0.2 , Figures $5(\mathrm{a})$ and $5(\mathrm{~b})$ show the relationship between replenishment parameters and the worst-case bullwhip $W_{i}^{2}$. For a specified $\alpha^{\text {wip }}$, the bullwhip effect will be very serious if we increase the value of $\alpha^{i}$. In contrast, for a specified $\alpha^{i}$, the relationship between $\alpha^{\text {wip }}$ and $W_{I}^{2}$ roughly follows a convex function. It means that, for fixed $\alpha^{i}$, there exists a optimal $\alpha^{w i p}$ to dampen production fluctuations. Figure 5(c) shows the impact of parameter $G$ on the bullwhip metric $W_{I}^{2}$. It has been emphasized that the parameter $G$ can be used to adjust the customer service level of the retailer. A large $G$ produces a high reorder point and thus brings extra inventory cost. However, Figure 5(c) indicates that increasing the value of $G$ will cause serious production fluctuations. Figure 5(d) shows the impact of demand forecasting parameter $\theta_{f}$ on the robust performance. It is quite clear to see that a large value of $\theta_{f}$ causes higher bullwhip effect for unknown demand. This phenomenon can be intuitively explained by the forecasting algorithm (7); that is, a small $\theta_{f}$ smooths the demand signal. A large $\theta_{f}$ implies that demand forecast follows the 


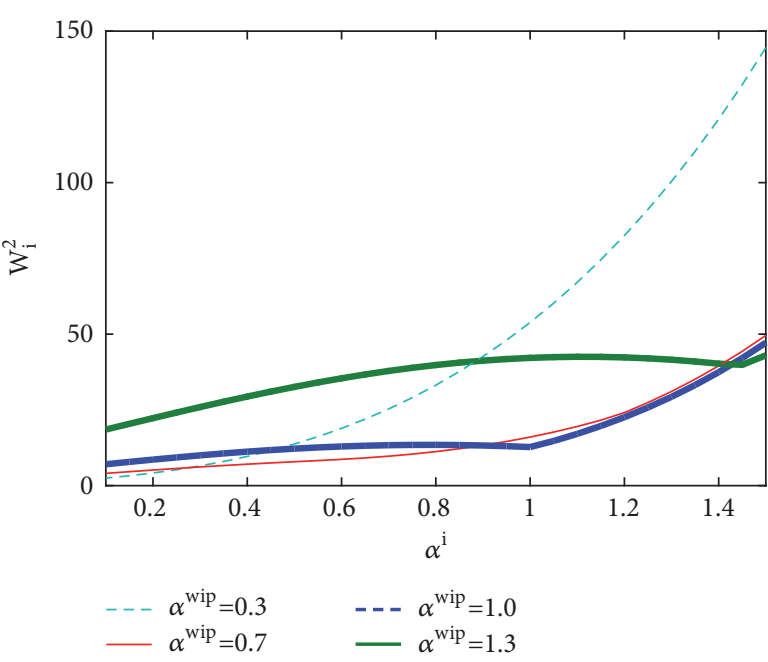

(a)

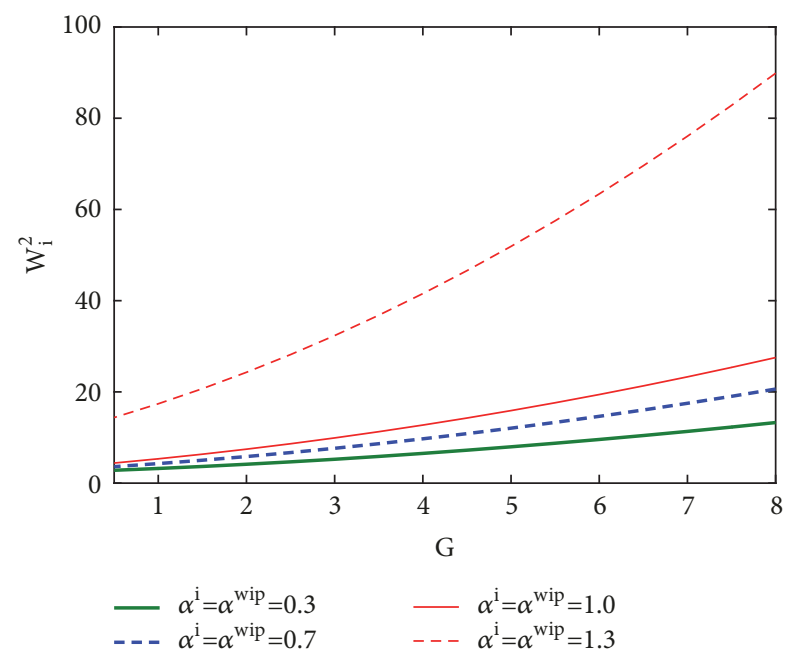

(c)

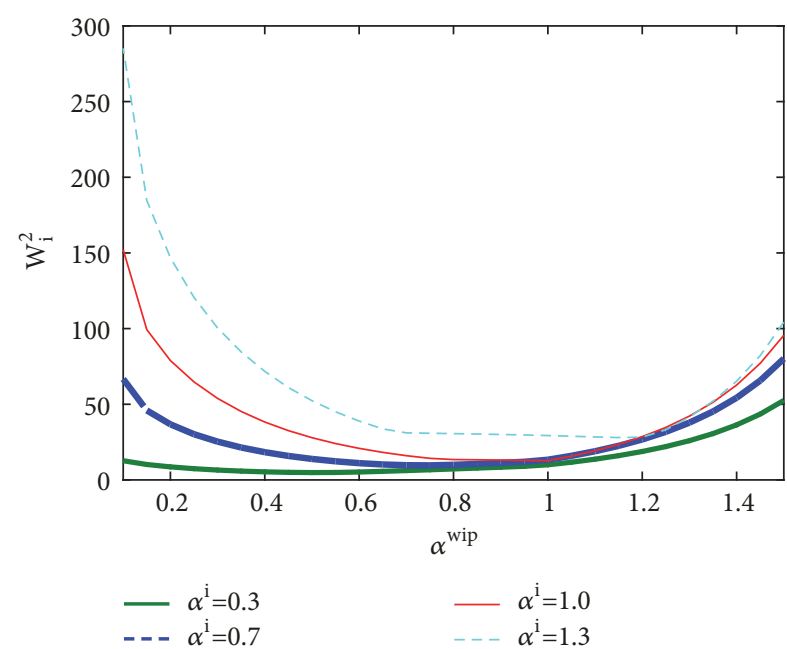

(b)

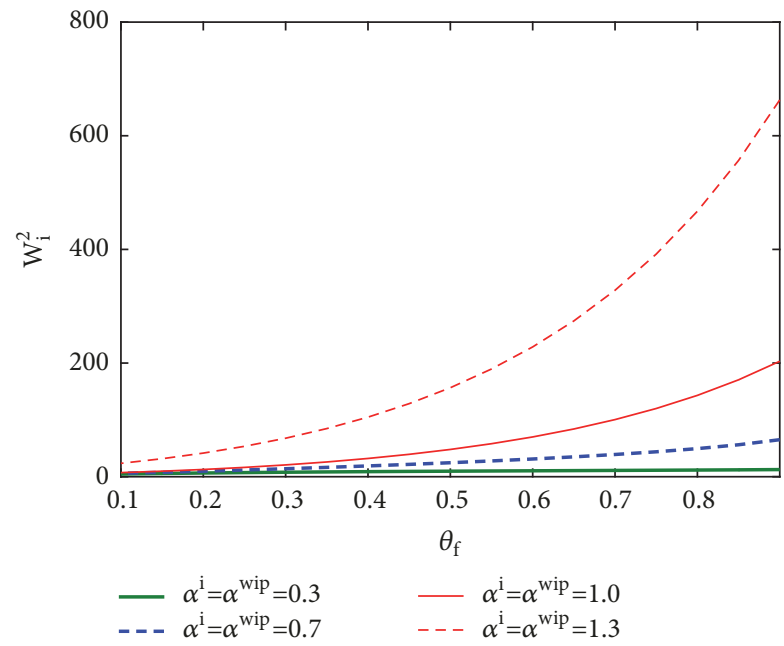

(d)

FIGURE 5: The impact of system parameters on the worst-case bullwhip.

historical demand immediately and causes high magnitude of production fluctuations.

\section{Discussions and Conclusions}

This paper has investigated the dynamics of a VMI supply chain system composed of one manufacturer and one retailer. To provide guidelines for the manufacturer to better manage the retailer's inventory, we firstly explored the fluctuation range of the retailer's inventory. We found that the retailer's inventory fluctuations are mainly influenced by demand characteristics, delivery frequency, lead time, and other parameters. Specifically, the magnitude of the retailer's inventory fluctuation is an increasing function of the parameter $\rho$, which reflects the delivery frequency. We notice that $\rho=1$ might cause poor customer service because in this situation, the reorder point might lose its ability to control the retailer's inventory. This result means that incorporating the parameter $\rho$ into the VMI model not only enhances the flexibility of decision-making, but also is useful in improving system performance. In practice, the upstream manufacturer should make replenishment decisions by optimizing inventory cost, transportation cost, and production cost simultaneously.

The shifting of the authority to manage the retailer's inventory and information sharing certainly affect the dynamics for the manufacturer. Based on the difference equations, we investigated the dynamical properties of the entire VMI supply chain system. It is interesting to demonstrate that, under the VMI program, the production process can be stabilized even if the retailer subsystem is unstable. This result seems to be favorable for the manufacturer; however, the retailer will face serious shortages because the manufacturer fails to satisfy the demand of the retailer. Until present, the majority of the literature on the dynamics of the VMI supply chain system is limited to simulation studies. For example, Disney et al. [53] showed that, under the VMI program, the manufacturer can choose a large delivery frequency to achieve economical scale without causing serious production 
fluctuations. It means that production cost and transportation cost can be reduced simultaneously, which is one of the advantages of VMI. In this paper, we confirm this result through theoretical analysis. However, we emphasize that managers should be cautious on determining the delivery frequency used to satisfy the retailer's demand. Although a high delivery frequency might be helpful in reducing transportation cost, the inventory cost of the retailer and the manufacturer can increase and the customer service level of the retailer can be very poor.

Based on the difference equations, we also studied the stability of the VMI supply chain. Stability is a fundamental problem for production smoothing. Although the production decision of the manufacturer is based on system inventory, we found that the stability condition of the VMI supply chain is very similar to a one-echelon production and inventory control system, as has been studied in Wei et al. [21]. To simplify the selection of replenishment parameters, we also obtained the stable region independent of the production lead time. Furthermore, we found that the stable region independent of the production lead time approximates the precise stable region as the production lead time increases. We further also studied production smoothing in terms of the worst-case bullwhip effect of the VMI supply chain system for unknown demand based on a transfer function method. Based on the transfer function of the VMI supply chain system, we numerically validate the effectiveness of the robust metric.

It is worth noting that this paper focuses on a VMI supply chain with a relatively simple structure and specific assumptions. In practice, the VMI program can be implemented for multiple supplier systems or distribution networks. In addition, a realistic supply chain system must be nonlinear [54]; thus incorporating nonlinearities into VMI models is a significant problem deserving future research.

\section{Data Availability}

The paper is focused on modeling and simulation. All the data is generated by simulation experiments with different parameter settings or available from the corresponding author upon request.

\section{Conflicts of Interest}

The authors declare that there are no conflicts of interest regarding the publication of this paper

\section{Acknowledgments}

This work was supported by the National Natural Science Foundation of China (nos. 71401181 and 71701213) and the MOE (Ministry of Education in China) Project of Humanities and Social Sciences (nos. 14YJC630136 and 15YJC630008).

\section{References}

[1] K. Govindan, "Vendor-managed inventory: A review based on dimensions," International Journal of Production Research, vol. 51, no. 13, pp. 3808-3835, 2013.
[2] A. Angulo, H. Nachtmann, and M. A. Waller, "Supply chain information sharing in a vendor managed inventory partnership," Journal of Business Logistics, vol. 25, no. 1, pp. 101-120, 2004.

[3] J.-Y. Lee, R. K. Cho, and S.-K. Paik, "Supply chain coordination in vendor-managed inventory systems with stockout-cost sharing under limited storage capacity," European Journal of Operational Research, vol. 248, no. 1, pp. 95-106, 2016.

[4] S. Çetinkaya and C.-Y. Lee, "Stock replenishment and shipment scheduling for vendor-managed inventory systems," Management Science, vol. 46, no. 2, pp. 217-232, 2000.

[5] P. Danese, "Beyond Vendor Managed Inventory: the Glaxosmithkline Case," Supply Chain Forum: An International Journal, vol. 5, no. 2, pp. 32-40, 2004.

[6] E. Watson, "Nestle switches to vendor managed inventory with tesco," Food Manufacture, vol. 80, no. 8, p. 20, 2008.

[7] V. A. Micheau, "How boeing and alcoa implemented a successful vendor managed inventory program," The Journal of Business Forecasting, vol. 24, no. 1, p. 17, 2005.

[8] J. H. Bookbinder, M. Gumus, and E. M. Jewkes, "Calculating the benefits of vendor managed inventory in a manufacturerretailer system," International Journal of Production Research, vol. 48, no. 19, pp. 5549-5571, 2010.

[9] X. Lu, J. Shang, S.-y. Wu, G. . Hegde, L. Vargas, and D. Zhao, "Impacts of supplier hubris on inventory decisions and green manufacturing endeavors," European Journal of Operational Research, vol. 245, no. 1, pp. 121-132, 2015.

[10] S. Birim and C. Sofyalioglu, "Evaluating vendor managed inventory systems: how incentives can benefit supply chain partners," Journal of Business Economics and Management, vol. 18, no. 1, pp. 163-179, 2017.

[11] J. Ru, R. Shi, and J. Zhang, "When Does A Supply Chain Member Benefit from Vendor-Managed Inventory?" Production Engineering Research and Development, vol. 27, no. 5, pp. 807-821, 2018.

[12] S. M. Disney and D. R. Towill, "The effect of vendor managed inventory (VMI) dynamics on the Bullwhip Effect in supply chains," International Journal of Production Economics, vol. 85, no. 2, pp. 199-215, 2003.

[13] M. Waller, M. E. Johnson, and T. Davis, "Vendor-managed inventory in the retail supply chain," Journal of Business Logistics, vol. 20, no. 1, p. 183, 1999.

[14] M. J. Fry, R. Kapuscinski, and T. L. Olsen, "Coordinating Production and Delivery under a (z, Z)-Type Vendor-Managed Inventory Contract," Manufacturing and Service Operations Management, vol. 3, no. 2, pp. 151-173, 2001.

[15] M. Ben-Daya, E. Hassini, M. Hariga, and M. M. AlDurgam, "Consignment and vendor managed inventory in single-vendor multiple buyers supply chains," International Journal of Production Research, vol. 51, no. 5, pp. 1347-1365, 2013.

[16] L. C. Coelho and G. Laporte, "An optimised target-level inventory replenishment policy for vendor-managed inventory systems," International Journal of Production Research, vol. 53, no. 12, pp. 3651-3660, 2015.

[17] A. A. Taleizadeh, M. Noori-Daryan, and L. E. CárdenasBarrón, "Joint optimization of price, replenishment frequency, replenishment cycle and production rate in vendor managed inventory system with deteriorating items," International Journal of Production Economics, vol. 159, pp. 285-295, 2015.

[18] J. Cai, P. R. Tadikamalla, J. Shang, and G. Huang, "Optimal inventory decisions under vendor managed inventory: substitution effects and replenishment tactics," Applied Mathematical 
Modelling: Simulation and Computation for Engineering and Environmental Systems, vol. 43, pp. 611-629, 2017.

[19] M. Khan, M. Y. Jaber, S. Zanoni, and L. Zavanella, "Vendor managed inventory with consignment stock agreement for a supply chain with defective items," Applied Mathematical Modelling, vol. 40, no. 15-16, pp. 7102-7114, 2016.

[20] D. E. Cantor and E. Katok, "Production smoothing in a serial supply chain: A laboratory investigation," Transportation Research Part E: Logistics and Transportation Review, vol. 48, no. 4, pp. 781-794, 2012.

[21] Y. C. Wei, H. W. Wang, and C. Qi, "On the stability and bullwhip effect of a production and inventory control system," International Journal of Production Research, vol. 51, no. 1, pp. 154-171, 2013.

[22] Y. C. Wei, H. W. Wang, and C. Qi, "The impact of stockdependent demand on supply chain dynamics," Applied Mathematical Modelling, vol. 37, no. 18-19, pp. 8348-8362, 2013.

[23] H. L. Lee, V. Padmanabhan, and S. Whang, "Information distortion in a supply chain: the bullwhip effect," Management Science, vol. 43, no. 4, pp. 546-558, 1997.

[24] F. Chen, Y. Wei, and H. Wang, "A heuristic based batching and assigning method for online customer orders," Flexible Services and Manufacturing Journal, pp. 1-46, 2017.

[25] X. Wang and S. M. Disney, "The bullwhip effect: progress, trends and directions," European Journal of Operational Research, vol. 250, no. 3, pp. 691-701, 2016.

[26] V. Giard and M. Sali, "The bullwhip effect in supply chains: A study of contingent and incomplete literature," International Journal of Production Research, vol. 51, no. 13, pp. 3880-3893, 2013.

[27] M. Udenio, E. Vatamidou, J. C. Fransoo, and N. Dellaert, "Behavioral causes of the bullwhip effect: An analysis using linear control theory," IISE Transactions, vol. 49, no. 10, pp. 980$1000,2017$.

[28] Y. Wei, F. Chen, and F. Xiong, "Dynamic Complexities in a Supply Chain System with Lateral Transshipments," Complexity, vol. 2018, Article ID 3959141, 15 pages, 2018.

[29] G. Huang, J. Wang, C. Chen, C. Guo, and B. Zhu, "System resilience enhancement: Smart grid and beyond," Frontiers of Engineering Management, vol. 4, no. 3, p. 271, 2017.

[30] S. M. Disney and D. R. Towill, "A discrete transfer function model to determine the dynamic stability of a vendor managed inventory supply chain," International Journal of Production Research, vol. 40, no. 1, pp. 179-204, 2002.

[31] B. Brunaud and I. E. Grossmann, "Perspectives in multilevel decision-making in the process industry," Frontiers of Engineering Management, vol. 4, no. 3, p. 256, 2017.

[32] G. Cachon and M. Fisher, "Campbell soup's continuous replenishment program: Evaluation and enhanced inventory decision rules," Production Engineering Research and Development, vol. 6, no. 3, pp. 266-276, 1997.

[33] Y. Yao and M. Dresner, "The inventory value of information sharing, continuous replenishment, and vendor-managed inventory," Transportation Research Part E: Logistics and Transportation Review, vol. 44, no. 3, pp. 361-378, 2008.

[34] J.-H. Kang and Y.-D. Kim, "Coordination of inventory and transportation managements in a two-level supply chain," International Journal of Production Economics, vol. 123, no. 1, pp. 137-145, 2010.

[35] S. Çetinkaya, E. Tekin, and C. Lee, "A stochastic model for joint inventory and outbound shipment decisions," Institute of
Industrial Engineers (IIE). IIE Transactions, vol. 40, no. 3, pp. 324-340, 2008.

[36] Y. Dong, M. Dresner, and Y. Yao, "Beyond information sharing: An empirical analysis of vendor-managed inventory," Production Engineering Research and Development, vol. 23, no. 5, pp. 817-828, 2014.

[37] Y. Yao, P. T. Evers, and M. E. Dresner, "Supply chain integration in vendor-managed inventory," Decision Support Systems, vol. 43, no. 2, pp. 663-674, 2007.

[38] M. Nagarajan and S. Rajagopalan, "Contracting under vendor managed inventory systems using holding cost subsidies," Production Engineering Research and Development, vol. 17, no. 2, pp. 200-210, 2008.

[39] J.-Y. Lee and R. K. Cho, "Contracting for vendor-managed inventory with consignment stock and stockout-cost sharing," International Journal of Production Economics, vol. 151, pp. 158173, 2014.

[40] B. K. Mishra and S. Raghunathan, "Retailer- vs. Vendormanaged inventory and brand competition," Management Science, vol. 50, no. 4, pp. 445-457, 2004.

[41] W. Pan and K. C. So, "Component procurement strategies in decentralized assembly systems under supply uncertainty," Institute of Industrial Engineers (IIE). IIE Transactions, vol. 48, no. 3, pp. 267-282, 2016.

[42] G. Kannan, M. C. Grigore, K. Devika, and A. Senthilkumar, "An analysis of the general benefits of a centralised VMI system based on the EOQ model," International Journal of Production Research, vol. 51, no. 1, pp. 172-188, 2013.

[43] A. Diabat, "Hybrid algorithm for a vendor managed inventory system in a two-echelon supply chain," European Journal of Operational Research, vol. 238, no. 1, pp. 114-121, 2014.

[44] M. Hariga, M. Gumus, and A. Daghfous, "Storage constrained vendor managed inventory models with unequal shipment frequencies," Omega , vol. 48, pp. 94-106, 2014.

[45] A. Mateen, A. K. Chatterjee, and S. Mitra, "VMI for singlevendor multi-retailer supply chains under stochastic demand," Computers \& Industrial Engineering, vol. 79, pp. 95-102, 2015.

[46] G. P. Cachon, T. Randall, and G. M. Schmidt, "In search of the bullwhip effect," Manufacturing \& Service Operations Management, vol. 9, no. 4, pp. 457-479, 2007.

[47] E. I. Jury, Theory and Application of the z-Transform Method, Wiley, 1964.

[48] C. R. Phillips and N. T. Nagle, IEEE Transactions on Systems, Man, and Cybernetics, Prentice Hall Press, 2007.

[49] C. S. Lalwani, S. M. Disney, and D. R. Towill, "Controllable, observable and stable state space representations of a generalized order-up-to policy," International Journal of Production Economics, vol. 101, no. 1, pp. 172-184, 2006.

[50] J. Dejonckheere, S. M. Disney, M. R. Lambrecht, and D. R. Towill, "Measuring and avoiding the bullwhip effect: a control theoretic approach," European Journal of Operational Research, vol. 147, no. 3, pp. 567-590, 2003.

[51] Y. Wang, R. Shou, L. H. Lee, and E. P. Chew, "A case study on sample average approximation method for stochastic supply chain network design problem," Frontiers of Engineering Management, vol. 4, no. 3, p. 338, 2017.

[52] Y. Ouyang, "The effect of information sharing on supply chain stability and the bullwhip effect," European Journal of Operational Research, vol. 182, no. 3, pp. 1107-1121, 2007. 
[53] S. M. Disney, M. M. Naim, and A. Potter, "Assessing the impact of e-business on supply chain dynamics," International Journal of Production Economics, vol. 89, no. 2, pp. 109-118, 2004.

[54] J. Chang and L. Zhao, "Complexity analysis of dynamic cooperative game models for supply chain with the remanufactured products," Discrete Dynamics in Nature and Society, Art. ID 9246934, 10 pages, 2018. 


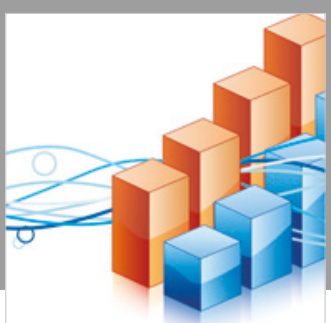

Advances in

Operations Research

\section{-n-m}
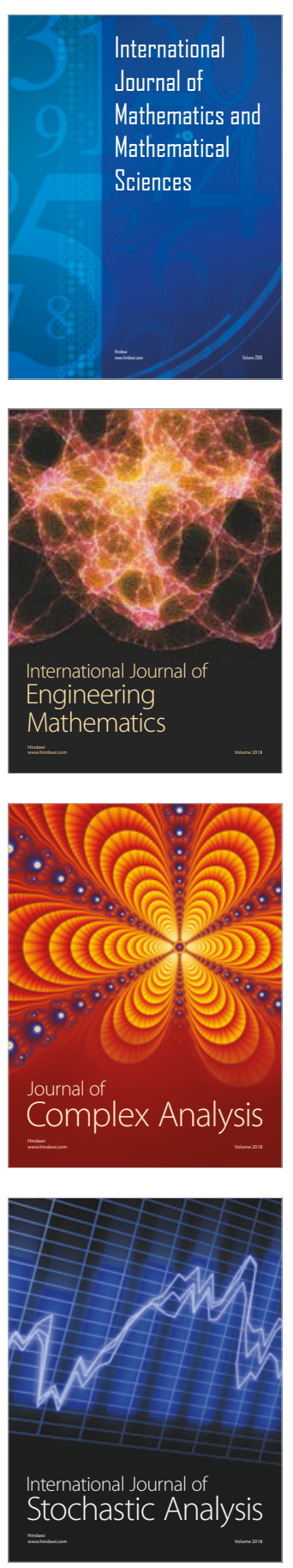
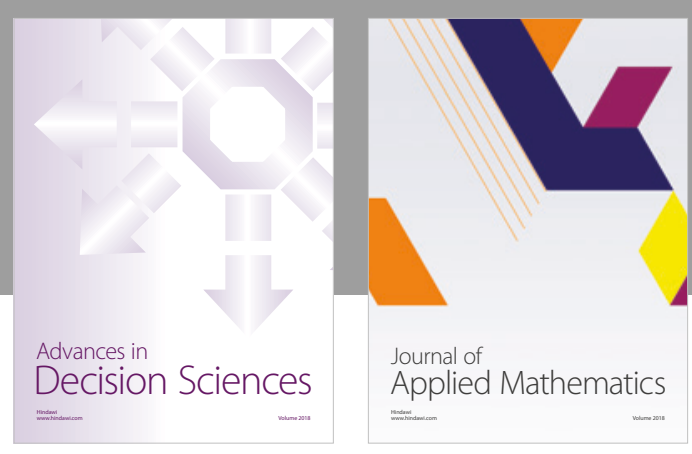

Journal of

Applied Mathematics
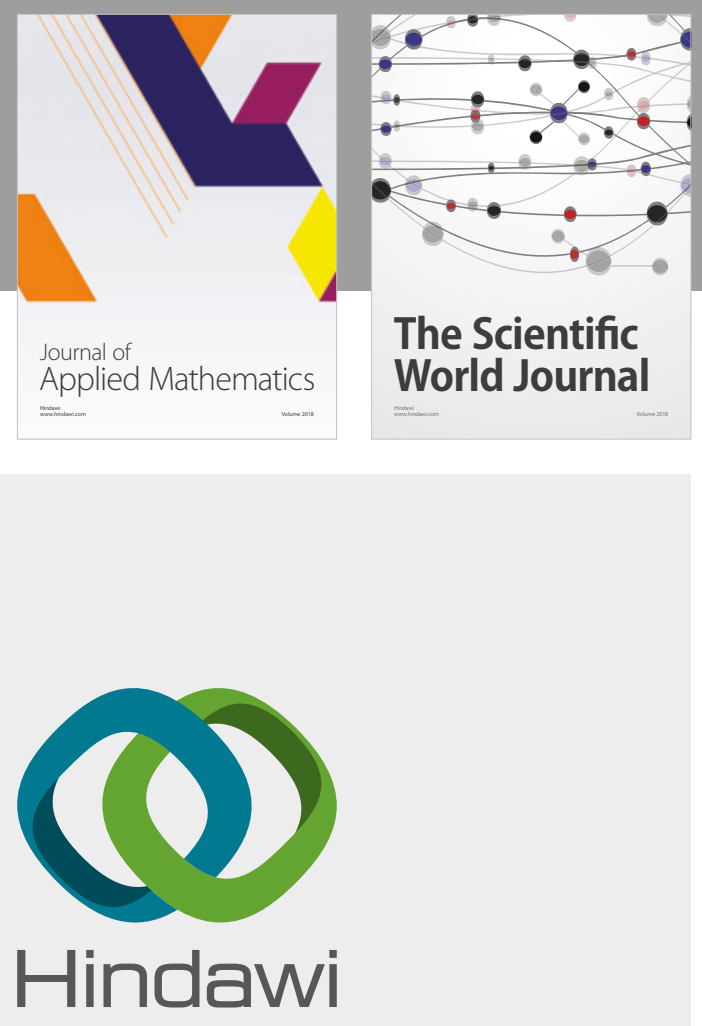

Submit your manuscripts at

www.hindawi.com

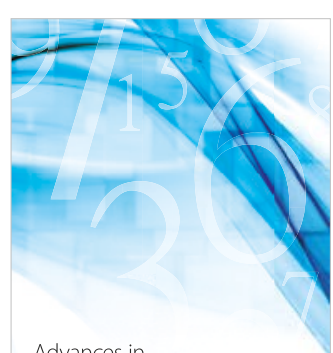

Advances in
Numerical Analysis
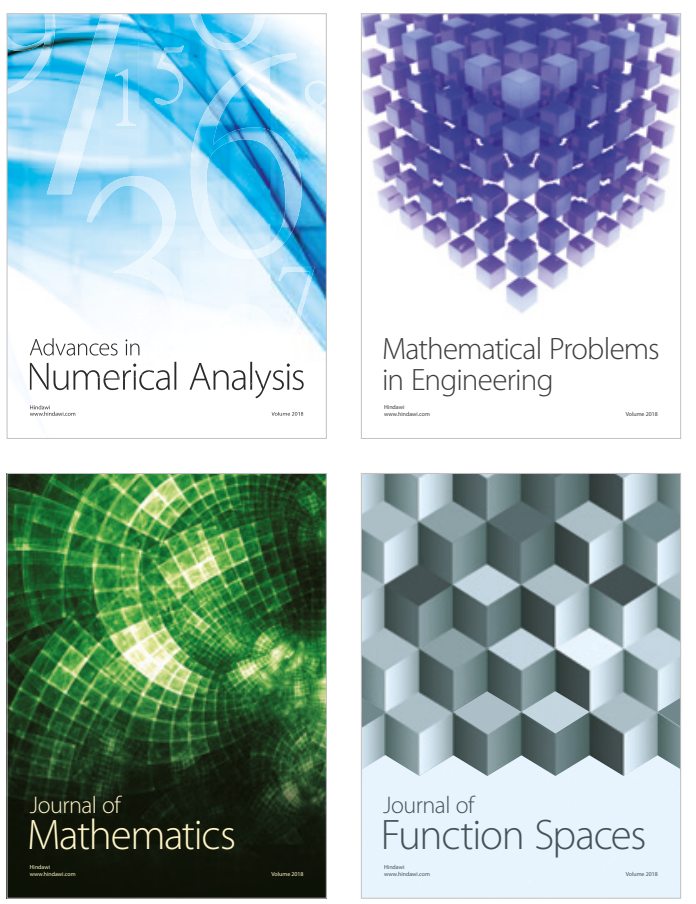

Mathematical Problems in Engineering

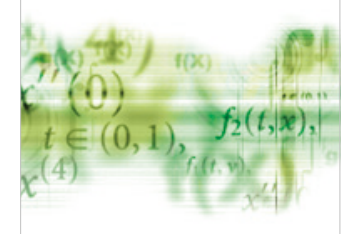

International Journal of

Differential Equations

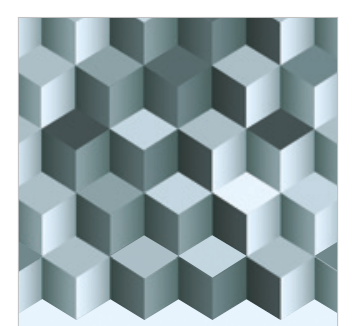

Journal of

Function Spaces

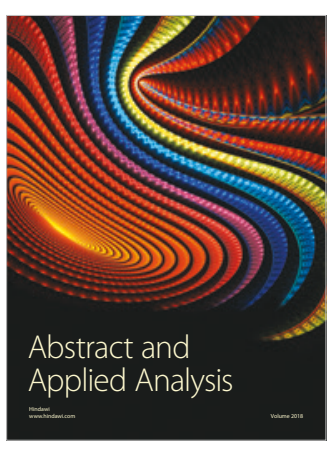

The Scientific

World Journal

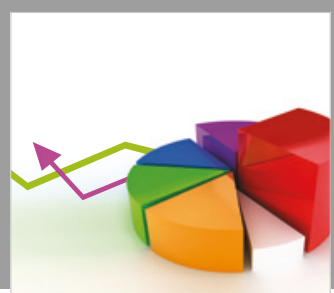

Journal of

Probability and Statistics
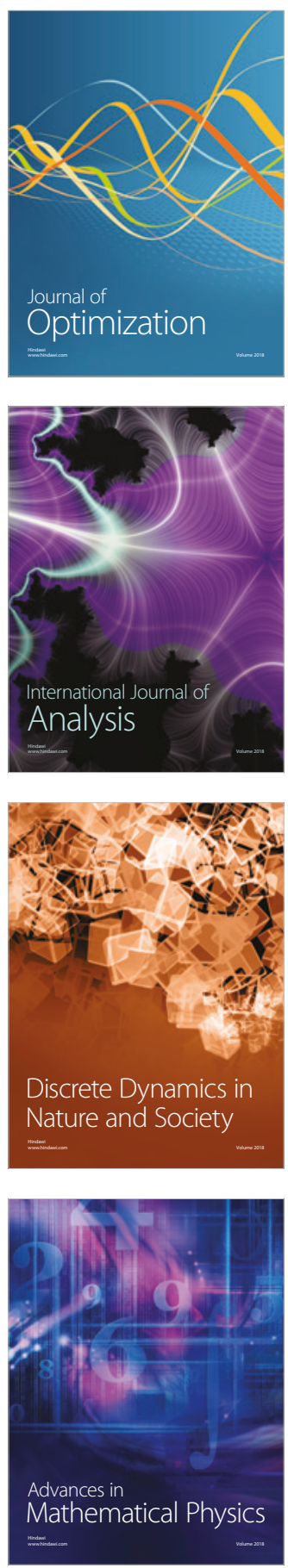